\title{
QCD as High Energy Limit of the Scalar Strong Interaction Hadron Theory
}

\author{
F. C. Hoh \\ Dragarbrunnsg, 55C, 75320 Uppsala, Sweden \\ Email: hoh@telia.com
}

Received June 24, 2013; revised July 26, 2013; accepted August 22, 2013

Copyright (C) 2013 F. C. Hoh. This is an open access article distributed under the Creative Commons Attribution License, which permits unrestricted use, distribution, and reproduction in any medium, provided the original work is properly cited.

\begin{abstract}
This paper is an extension of the book of reference [1] below. QCD Lagrangian is derived from the same equations of motion for quarks used to construct the equations of motion for mesons and baryons in the scalar strong interaction hadron theory that accounts for many basic low energy data not covered by QCD. At high energies, the energetic quarks in a hadron can be far from each other and approximately free. Each quark is associated with a vector in an internal space characterizing its mass and charge. These spaces are interchangeable and provide a new symmetry equivalent to color symmetry in QCD. A quark in a meson has two "colors" and in a baryon three "colors"; the $\beta$ function of QCD is $61 \%-92 \%$ greater in high energy interactions leading to baryons than that to mesons. This function enters the measurable running coupling constant and this prediction is testable against experiment. QCD, successful at high energies, is thus reconciled with the scalar strong interaction hadron theory and both complement each other.
\end{abstract}

Keywords: QCD at High Energies; Scalar Strong Interaction; Internal Symmetry

\section{Introduction}

The scalar strong interaction hadron theory $[1,2]$ can account, to a limited extent of varying accuracy, low energy hadronic phenomena of basic importance. The high energy end has been left out. Quantum Chromodynamics (QCD) on the other hand, has proven to be successful at high energies but can basically not account for low energy phenomena.

The situation may be summarized in Table 1, which includes the established QED as reference.

In the following, references of the form $(x, y, z)$, Section $x . y, \S x . y . z$, pxyz, and Table $\mathrm{x} . \mathrm{y}$ refer to those in [1].

QCD is a quantum field theory for "colored" quarks based on the QCD Lagrangian [3 Reviews.../Standard Model.../Quantum Chromodynamics, Equations $(9.1,2)]$. The interquark force is of "color" vectorial nature. However, the equations of motion at the quantum mechanical level obtained from the QCD Lagrangian are in terms of unobservable "colored" quarks and are of no use at low energies. This theory has proven to be successful at high energies.

The equations of motion for ground state mesons (2.4.2) and for baryons (9.3.11) form the basis of the present scalar strong interaction hadron theory. There is no quark wave function in these equations and the interquark force is of scalar nature. These equations and the corresponding Lagrangians can be converted into each other. The theory remains largely at the quantum mechanical level and, as mentioned in $\S 6.4 .4$, has not been quantized.

The wave functions for mesons in (2.4.2) and for baryons in (9.3.11) have been solved for hadrons at rest. In motion, only dimensional estimates for pseudoscalar meson are given in Section 3.5. Feyman propagator for these hadrons is thus not known and Feynman diagrams in an eventual quantized version cannot be evaluated. More basically, the free hadron wave functions in (2.4.2) and (9.3.11) contain via (3.2.3a) in addition to the laboratory coordinate $X$, also the relative coordinate $x$ as well as the internal coordinate $z$ and $u$. The latter ones have no correspondence in classical mechanics and the usual transition rule to quantum mechanics is insufficient. This agrees with the known fact that nonlocal thoeries cannot be quantized.

\section{Genaralized Equations of Motion for Quarks}

The starting point of the scalar strong interaction meson theory $[1,2]$ is a quark $A$ at space-time point $x_{I}$ with flavor $p$ interacting scalarly with an antiquark $B$ at point $x_{I I}$ having antiflavor $r$ described by 
Table 1. Key ingredients in QED, QCD and SSI (scalar strong interaction hadron theory) for comparison.

\begin{tabular}{|c|c|c|c|}
\hline Scalar Strong Int-SSI & QCD & QED & \\
\hline Nonlocal theory Cannot be quantized & $\begin{array}{l}\text { Loop diagrams } \\
\text { Renormalization } \\
\text { diff. gauges } \\
\text { Asymtotic freedom-1st order } \\
\text { nonAbelian self coupling }\end{array}$ & $\begin{array}{l}\text { Loop diagrams } \\
\text { Renormalization }\end{array}$ & QUANTUM FIELD THEORY \\
\hline $\begin{array}{l}\text { At high energies, the quarks are nearly free. The } \\
\text { different internal spaces for quarks in SSI provide } \\
\text { a new degree of freedom similar to color in QCD, } \\
\text { which can largely be taken over (this paper) }\end{array}$ & $\begin{array}{l}\text { Feynman Rules: }(\mathrm{FR}) \\
\text { quark } \approx \\
\text { gluon } \approx \\
\text { Ghost-nonAbelian self coupling }\end{array}$ & $\begin{array}{c}\text { Feynman Rules: (FR) } \\
\text { lepton } \\
\text { photon }\end{array}$ & \\
\hline $\begin{array}{l}\text { Predictive power: } \\
\text { Similar to QCD's }\end{array}$ & $\begin{array}{l}\text { Predictive power: } \\
\text { Good }\end{array}$ & $\begin{array}{l}\text { Predictive power: } \\
\text { Highly accurate }\end{array}$ & $\begin{array}{l}\text { Higher energies, Higher orders in } \\
\text { coupling constant }\end{array}$ \\
\hline $\begin{array}{c}A_{\mu v}^{1}=\partial_{\mu} A_{v}^{1}-\partial_{v} A_{\mu}^{1}+q \varepsilon^{l m n} A_{\mu}^{m} A_{v}^{n} \\
A_{\mu}^{1}=\text { like "gluon" }\end{array}$ & $\begin{array}{c}F_{\mu v}^{1}=\partial_{\mu} V_{v}^{1}-\partial_{\nu} V_{\mu}^{1}+g f^{l m n} V_{\mu}^{m} V_{v}^{n} \\
V_{\mu}^{1}=\text { gluon }\end{array}$ & $\begin{array}{c}F_{\mu v}=\partial_{\mu} A_{v}-\partial_{v} A_{\mu} \\
A_{\mu}=\text { photon }\end{array}$ & QUANTUM MECHANICS \\
\hline $\begin{array}{l}\text { SSI Lagrangians } \\
\uparrow \\
\text { Meson Equation of motion } \\
\text { (akin to } \lambda \phi^{4} \text { theory) } \\
\text { Baryon Equation of motion }\end{array}$ & $\begin{array}{c}\text { QCD Lagrangian } \\
\text { ( } \uparrow \text { FR) } \\
\text { does not yield } \\
\text { useful Equation of motion }\end{array}$ & $\begin{array}{c}\text { QED Lagrangian } \\
\uparrow \\
\text { Dirac Equation ( } \uparrow \mathrm{FR}) \\
\text { Maxwell Equation ( } \uparrow \mathrm{FR})\end{array}$ & \\
\hline $\begin{array}{l}\text { Predictive power: Limited but of basic nature, } \\
\text { Ok presently }\end{array}$ & $\begin{array}{l}\text { Predictive power: } \\
\text { Nearly none }\end{array}$ & $\begin{array}{l}\text { Predictive power: } \\
\text { Firmly established }\end{array}$ & $\begin{array}{c}\text { Low energies, } \\
\text { 0th, 1st order in coupling constant }\end{array}$ \\
\hline
\end{tabular}

$$
\begin{aligned}
& {\left[-i \gamma^{\mu}\left(\frac{\partial}{\partial x_{I}^{\mu}}+i q_{o p}(z) A_{\mu}\left(x_{I}\right)\right)\right.} \\
& \left.+m_{1 o p}(z)-V_{S B}\left(x_{I}\right)\right] \psi_{A}\left(x_{I}\right) z_{I}^{p}=0 \\
& {\left[-i \gamma^{\mu}\left(\frac{\partial}{\partial x_{I I}^{\mu}}+i q_{o p}(z) A_{\mu}\left(x_{I I}\right)\right)\right.} \\
& \left.+m_{1 o p}(z)-V_{S A}\left(x_{I I}\right)\right] \psi_{B}\left(x_{I I}\right) z_{I I r}=0
\end{aligned}
$$

In (1) and (2), following the notations of (A1-A5), $\psi$ is usual Dirac bispinor and $V_{S B}\left(x_{I}\right)$ is the scalar potential (2.1.2) emanating from $B$ acting on $A$ and vice versa (2.1.4) for $V_{S A}\left(x_{I I}\right) . z^{p}$ are $p$ complex variables originally providing a point field for implementing $\mathrm{SU}(p)$ transformations [4] and $z_{p}=\left(z^{p}\right) *$ in (2.3.4). They [2, Section5] acquire here a more physical role as eigenfunctions of the mass operator [2 (9.6a)], (2.3.26)

$$
m_{1 o p}(z)=m_{q}\left(z_{I}^{q} \frac{\partial}{\partial z_{I}^{q}}+z_{I q} \frac{\partial}{\partial z_{I q}}+z_{I I}^{q} \frac{\partial}{\partial z_{I I}^{q}}+z_{I I q} \frac{\partial}{\partial z_{I I q}}\right)
$$

as well as the charge operator

$$
\begin{aligned}
q_{o p}(z) & =q_{r}\left(z_{I}^{r} \frac{\partial}{\partial z_{I}^{r}}-z_{I r} \frac{\partial}{\partial z_{I r}}+z_{I I}^{r} \frac{\partial}{\partial z_{I I}^{r}}+z_{I I r} \frac{\partial}{\partial z_{I I r}}\right) \\
& =-q_{o p}^{*}(z)
\end{aligned}
$$

generalized from (2.3.14). Repeated indices are summed over. Acting upon $z^{p}$ in (1), (3) and (4) produce the quark mass $m_{p}$ and charge $q_{p}$, respectively, as eigenvalues. The $A_{\mu}$ 's are the associated electromagnetic fields. $\psi_{A}\left(x_{I}\right) z_{I}^{p}$ is regarded as the total wave function for quark $A$ and
$\psi_{B}\left(x_{I I}\right) z_{I I r}$ that of the antiquark $B . z_{I}$ and $z_{I I}$ refer to different internal or flavor spaces in Section 2.1 and are as distinct as $x_{I I}$ and $x_{I}$. These $z$ 's, like the relative coordinates $x=x_{I I}-x_{I}$, are "hidden varaiables" in $\S 2.3 .5$, p. 327. The above equations are taken as hypothetical, as free quark has not been observed, but are used to construct equations of motion for meson. For this purpose, (1) was originally written in van der Waerden's two spinor form (2.1.1a, 3a) via (2.3.11).

\section{Quark Confinement in Meson}

The so-constructed equations of motion have been solved for mesons at rest to account for a number of basic problems in the book [1]. After its publication, CP violations in neutral kaon decays have been substantially clarified [5].

The rest frame pseudoscalar meson is confined by the interquark potential $(3.2 .8 \mathrm{a}, 19,20)$ via the genealization (2.2.3),

$$
\begin{gathered}
V_{S A}\left(x_{I I}\right) V_{S B}\left(x_{I}\right) \rightarrow \Phi_{m}\left(x_{I}, x_{I I}\right) \rightarrow \Phi_{m}(\underline{x}) \\
=-\Phi_{c}(\underline{x})+\frac{d_{m}}{r}+d_{m 0} \\
\Phi_{c}(r \rightarrow \infty)=\frac{g_{s}^{4}}{2} r \int_{0}^{\infty} \mathrm{d} r^{\prime} r^{\prime 2}\left|\psi_{0}\left(r^{\prime}\right)\right|^{2}=\beta_{m 0} r
\end{gathered}
$$

where $r=\left|\underline{x}_{I I}-\underline{x}_{I}\right|=|\underline{x}|$ is the interquark distance, $d_{m}=$ $0.864 \mathrm{Gev}$ in (5.2.3), $d_{m 0}=0.24455 \mathrm{Gev}^{2}$ in Table 5.2, $g_{s}^{2}$ is the scalar strong quark-quark coupling in (2.2.1, $4)$, and $\Phi_{c}(\underline{x})$ the nonlinear (in $\psi$ ) confinement which provides linear confinement at large $r$. The meson wave fnnction $\psi_{0}(r)$ is formed from generalization of the 
product of $\psi_{A}\left(x_{I}\right)$ in (1) and $\psi_{B}\left(x_{I I}\right)$ in (2) according to (2.2.1), satisfies (3.2.10) and is given by (4.3.2)

$$
\psi_{0}(r)=\sqrt{\frac{d_{m}^{3}}{8 \pi \Omega}} \exp \left(-d_{m} r / 2\right)
$$

where $\Omega$ is a large normalization volume in the laboratory space $\underline{X}=\left(\underline{x}_{I I}+\underline{x}_{I}\right) / 2$. For a free meson, $\Omega \rightarrow \infty$ and (7) and (6) $\rightarrow 0$ and the quarks are confined by $d_{m} / r$ in (5) only. From (7), the size of the meson is about $2 \mathrm{fm}$ in (4.7.3); the quarks are tightly bound.

If the same meson is moving, Section 3.5 shows that its wave function is of the plane wave form

$\exp \left(-i E X^{0}+i \underline{K X}\right)\left(\psi_{0}(\underline{x}), \psi(\underline{x})\right)$, where $E$ is the energy and $\underline{K}$ the momentum, and is a four vector in relative space. $\psi_{0}$ is the time and large component and the vector part $\Psi$ makes up the spatial and small components. Equations governing these components have not been solved. In addition, the free meson wave function is seen to be nonlocal and can therefore not be quantized.

For small $K$, however, approximate forms of $\psi_{0}$ and $\psi$ have been estimated using dimensional approximations and $\psi \propto K$, shown in $\$ 3.5 .3,4$.

As is discussed in Section 4.5, the above plane wave form is distorted and the corresponding quantity playing the role of $\Omega$ no longer $\rightarrow \infty$ when the meson is interacting with another particle. Hence, the wave function becomes finite and the linear confinment (6) is called into action in (5); the quarks are always confined.

At higher energies, the quarks also become energetic and the interquark distance $r$ is expected to be large so that the confining term $d_{m} / r$ in (5) becomes small. In this case, quarks are no longer tightly bound and the nonlinear confinement $\Phi_{c}$ in (6) can still be weak over a large range of $r$ for large enough $\Omega$ type of volumes. In this $r$ range, $\Phi_{m}$ in (5) is small and quarks may be considered as approximately free so that (1) and (2) with $V_{S B}, V_{S A} \rightarrow$ 0 via the left member of (5) are applicable.

\section{Internal Space Symmetry and Gauge Transformation}

In Section 12.8, on p. 272 at the end of the book, it was pointed out that "The internal degrees of freedom in form of the three internal coordinates $z_{I}, z_{I I}$ and $z_{I I I}$ of Section 9.3 can play some of the roles of the three colors in QCD...". This observation will now be pursued and developed. The two internal spaces $z_{I}$ and $z_{I I}$ in (1) and (2) are not observables and are interchangeable and thus provide a new symmetry analogous to color symmetry in QCD. Noting this, the total quark wave function

$\psi_{A}\left(x_{I}\right) z_{I}^{p}$ in (1) is generalized to a column matrix with two elements, one with $z_{I}$ the other $z_{I I}$. Consequently, the $\mathrm{U}(1)$ gauge field $A_{\mu}$ must also be generalized to an SU(2) gauge field. The so-generalized (1) and (2) read

$$
\begin{aligned}
& {\left[-i \gamma^{\mu}\left(\frac{\partial}{\partial x_{I}^{\mu}}+i q_{o p}(z) \overline{\underline{\sigma} \underline{A}_{\mu}}\left(x_{I}\right)\right)+m_{1 o p}(z)\right] \Psi_{A}^{p}=0} \\
& \Psi_{A}^{p}=\left(\begin{array}{l}
\psi_{A}\left(x_{I}\right) z_{I}^{p} \\
\psi_{A}\left(x_{I}\right) z_{I I}^{p}
\end{array}\right) \\
& {\left[-i \gamma^{\mu}\left(\frac{\partial}{\partial x_{I I}^{\mu}}-i q_{o p}(z)\left\{\bar{\sigma} \underline{A}_{\mu}\left(x_{I}\right)\right\}_{I \leftrightarrow I I}\right)+m_{1 o p}(z)\right] \Psi_{B r}=0} \\
& \Psi_{B r}=\left(\begin{array}{l}
\psi_{B}\left(x_{I I}\right) z_{I I r} \\
\psi_{B}\left(x_{I I}\right) z_{I r}
\end{array}\right)
\end{aligned}
$$

observing the definitions

$$
A=\left(\begin{array}{ll}
A_{11} & A_{12} \\
A_{21} & A_{22}
\end{array}\right), \quad \bar{A}=\left(\begin{array}{ll}
A_{11} z_{I}^{p} \partial / z_{I}^{p} & A_{12} z_{I}^{p} \partial / z_{I I}^{p} \\
A_{21} z_{I I}^{p} \partial / z_{I}^{p} & A_{22} z_{I I}^{p} \partial / z_{I I}^{p}
\end{array}\right)
$$

The stepping operators of the from $z_{I} \partial / \partial z_{I I}$ has been introduced earlier [6] in connection with the $W^{ \pm}$bosons. As the $\bar{A}$ type of matices in (8) and (9) operates on $z_{I}$ and $z_{I I}$,

$$
\begin{aligned}
& \bar{A}^{2}=\left(\begin{array}{ll}
A_{11} z_{I}^{p} \partial / z_{I}^{p} & A_{12} z_{I}^{p} \partial / z_{I I}^{p} \\
A_{21} z_{I I}^{p} \partial / z_{I}^{p} & A_{22} z_{I I}^{p} \partial / z_{I I}^{p}
\end{array}\right)^{2}=A \bar{A}=\overline{A^{2}}, \\
& \bar{A}^{n}=A^{n-1} \bar{A}=\overline{A^{n}}
\end{aligned}
$$

because the second order deivatives of the form $\partial^{2} / \partial z_{I}^{2}$, $\partial^{2} / \partial z_{I} \partial z_{I I}$ in (11) vanish. Therefore, all higher order deivatives in $\mathrm{z}_{I}$ and $z_{I I}$ also drop out. Also, $z_{I} \partial / \partial z_{I}$ and $z_{I I} \partial / \partial z_{I I}$ in $\bar{A}$ may be dropped without affecting the results.

Analogous to the usual field tensor [7 (18.8)], define the gauge field tensor

$$
\begin{aligned}
& \overline{\underline{\sigma} \underline{A}_{\mu v}}\left(x_{I}\right)=\frac{\partial}{\partial x_{I}^{\mu}} \overline{\underline{\sigma}} \underline{A}_{\mu}\left(x_{I}\right)-\frac{\partial}{\partial x_{I}^{v}} \overline{\underline{\sigma} \underline{A}_{\mu}}\left(x_{I}\right) \\
& +i q_{o p}(z)\left[\bar{\sigma} \underline{A}_{\mu}\left(x_{I}\right), \bar{\sigma} \underline{A}_{v}\left(x_{I}\right)\right]
\end{aligned}
$$

Let

$$
U_{2}\left(x_{I}\right)=\exp \left(i \underline{\sigma} \underline{\vartheta}\left(x_{I}\right)\right), \overline{U_{2}}\left(x_{I}\right)=\exp \left(i \overline{\bar{\sigma} \underline{\vartheta}}\left(x_{I}\right)\right)
$$

Under the $\mathrm{SU}(2)$ gauge transformations

$$
\begin{gathered}
\Psi_{A}^{p} \rightarrow \Psi_{A}^{\prime p}=\left(\begin{array}{l}
\psi_{A}\left(x_{I}\right)^{\prime} z_{I}^{p} \\
\psi_{A}\left(x_{I}\right)^{\prime} z_{I I}^{p}
\end{array}\right)=\overline{U_{2}}\left(x_{I}\right) \Psi_{A}^{p} \\
\overline{\bar{\sigma} \underline{A}_{\mu}}\left(x_{I}\right) \rightarrow \bar{\sigma} \underline{A}_{\mu}\left(x_{I}\right)^{\prime}=\overline{U_{2}}\left(x_{I}\right) \bar{\sigma} \underline{A}_{\mu}\left(x_{I}\right) \overline{U_{2}^{-1}}\left(x_{I}\right) \\
-\frac{i}{q_{o p}(z)} \overline{U_{2}}\left(x_{I}\right) \frac{\partial}{\partial x_{I}^{\mu}} \overline{U_{2}^{-1}}\left(x_{I}\right)
\end{gathered}
$$

(8) and (12) are invariant; these invariances are the same as the conventional ones without the $z$ 's in (13) and in (8) and (12), noting that (3) and (4) are invariant under $z_{I} \leftrightarrow$ 
$z_{I I}$ and can respectively be replaced by $m_{p}$ and $q_{p}$. $\bar{A}_{\mu}$ corresponds to the SU(2) gluons in QCD. There are only two degrees of freedom represented by $z_{I}$ and $z_{I I}$ corresponding to two colors for quarks in a meson.

\section{Derivation of QCD Lagrangian}

The quark Lagrangian density $L_{Q}$ is obtained by multiplying (8) from the left by $\bar{\Psi}_{A p}$ and integrating over the angles in $z_{I}^{p}$ and $z_{I I}^{p}$. Limiting ourselves to light quarks $u, d$ and $s$ corresponding respectively to flavor $p=$ 1,2 and 3, which have about the same quark mass $m_{p}$ in Table 5.2, one obtains

$$
\begin{aligned}
& \bar{\Psi}_{A p}=\left(\bar{\psi}_{A}\left(x_{I}\right) z_{I p}, \bar{\psi}_{A}\left(x_{I}\right) z_{I I p}\right) \\
& L_{Q}=\int \mathrm{d} v_{z_{I} p} \mathrm{~d} v_{z_{I I} p} \\
& \cdot\left\{\bar{\Psi}_{A p}\left[-i \gamma^{\mu}\left(\frac{\partial}{\partial x_{I}^{\mu}}+i q_{p} \bar{\sigma} \underline{A}_{\mu}\left(x_{I}\right)\right)+m_{p}\right] \Psi_{A}^{p}\right\} \\
& =C_{N}\left(\bar{\psi}_{A}\left(x_{I}\right), \bar{\psi}_{A}\left(x_{I}\right)\right) \\
& \cdot\left[-i \gamma^{\mu}\left(\frac{\partial}{\partial x_{I}^{\mu}}+i q_{p} \underline{\sigma}_{\mu}\left(x_{I}\right)\right)+m_{p}\right]\left(\begin{array}{l}
\psi_{A}\left(x_{I}\right) \\
\psi_{A}\left(x_{I}\right)
\end{array}\right), \\
& C_{N}=N_{p}^{2} \frac{\pi^{6}}{3}
\end{aligned}
$$

Here, $(2.3 .9,6 \mathrm{~b})$ have been employed and $N_{p}$ is a constant in (2.3.9). The quark charge $q_{p}$ becomes here an unrenormalized coupling constant.

The SU(2) "gluon" Lagrangian density $L_{G}$ is similarly obtained

$$
\begin{aligned}
& L_{G} \\
& =\int \mathrm{d} v_{z_{I} p} \mathrm{~d} v_{z_{I I} p}\left\{z_{K p}\left(-\frac{1}{2}\right) \operatorname{trace}\left[\overline{\underline{\sigma} \underline{A}_{\mu v}}\left(x_{I}\right) \overline{\underline{\sigma} \underline{A}_{\mu v}}\left(x_{I}\right)\right] z_{K}^{p}\right\} \\
& =C_{N}\left(-\frac{1}{2}\right) \operatorname{trace}\left[\underline{\sigma} \underline{A}_{\mu v}\left(x_{I}\right) \underline{\sigma}_{\mu v}\left(x_{I}\right)\right]
\end{aligned}
$$

where $K$ runs from $I$ to $I I$ and (4) has been used in (12). Note that quarks having different "colors" or $z_{K}$ 's have the same space time wave function in (15), as it should. Adding (15) and (16) yields the total "two color" Lagragian density for $p=1,2$ or 3 .

$$
L_{Q}+L_{G}=C_{N} L_{Q C D 2}
$$

The conventional QCD Lagragian density $L_{Q C D 2}[7,6$ line below (18.6)] for two colors is thus recovered.

The antiqauark Equation (9) can be treated analogously leading to the same results.

Baryon consists of three quarks and makes use of three of (1) associated with three internal spaces $z_{I}, z_{I I}$ and $z_{I I I}$ in (9.3.1) or three internal degrees of fredom or "colors". At rest, two quarks merge to form a diquark via (9.2.12) and (9.3.4) and there are only two "colors". The quarkdiquark confinement potential $(10.1 .6 \mathrm{a}, 8)$ differs from (5) in that $d_{b 2} r^{2}$ there provides confinement independent of the nonlinear confinement $\Phi_{b c}(\underline{x})$ there; the quark and the diquark are always confined irrespective whether there is another particle nearby.

When interacting with another particle at higher energies, the quarks also become energetic and the diquark is expected to break up so that there are three interquark distances instead of $r$. The corresponding form of $\Phi_{b}(\underline{x})$ is unknown but these three distances, like $r$ in high energy mesons, are expected to be large so that the three quarks may be regarded as approximately free in some ranges of the three distances. Again, the nonlinear confinement corresponding to $\Phi_{b c}(\underline{x})$, which provides cubic form of confinement at large $r$ in the quark-diquark configuration (10.2.5b), renders the quarks to be confined. In this case, the above treatment of quarks in meson can straightforwardly be extended to apply to quarks in baryons. The Pauli matrices $\underline{\sigma}$ above is replaced by the GellMann matrices $\underline{\lambda}$ and $\underline{\theta}$ has now eight components. The conventional $L_{Q C D 3}[7,6$ line below (18.6)] for three colors is recovered.

The whole development of $L_{Q C D 2}$ and $L_{Q C D 3}$, including quantization, choice of gauges, renormalization, Feynman rules, asymtotic freedom, etc can be taken over. The QCD beta function $\propto 11 C_{2}-2 n_{f}[7(18.146)]$ where $C_{2}$ is 3 for baryon and 2 for meson and the number of flavors $n_{f}$ is $2-5$. This function is thus $61 \%-92 \%$ greater in high energy interactions leading to baryons than that to mesons. As this function enters the measurable running coupling constant, this prediction should be testable against experiment.

This effect appears to be effective at large quark separations $r$ for confinement which is taken over by the scalar strong interaction confinement $d_{m} / r$ in (5) and $d_{b} / r$ in (10.1.6a) for small $r$.

In this way, QCD is reconciled with the scalar strong interaction hadron theory and complement each other; the former holds at high energies while the latter accounts for, so far to limited extent of basic nature, data at the low energy end of elementary particle theory. The intermediate energy range remains not covered.

\section{On Exact and Broken Symmetry}

The known symmetries, C, P, T, and electroweak SU(2) gauge, are all broken. Because they reside in space-time and isospace, the degrees of symmetry breaking can be measured. The SU(2) internal or "color" gauge symmetry here is however exact and cannot be broken. The associated gauge transformation act on two abstract, internal spaces $z_{I}$ and $z_{I I}$, which have been created artifcially [2 Section 5], Section 2.3 to accommodate different quark flavor vectors. These two spaces are identical in structure 
and there is no quantity in the formalism that distinguish them from each other, i.e., can break this $z_{I} \leftrightarrow z_{I I}$ symmetry. Even if some such quantity will appear later, it cannot be measured to determine the degree of symmetry breaking because $z_{I}$ and $z_{I I}$ are unobservable "hidden" variables mentioned below (4). Such a symmetry breaking would appear as nonexisting. As the "gluons" in $(8,9)$ depend upon the unobservable quark coordinates $x_{I}$ and $x_{I I}$ as well as $z_{I}$ and $z_{I I}$, they can also not be observed.

\section{Conclusion}

QCD has hitherto been based upon the assumption that a quark has three colors. This has its root in the Pauli exclusion principle. This principle has been confirmed for freely observable fermions; but quarks are not freely observable and hence do not have to obey this principle and the above assumptions become ad-hoc. In the scalar strong interaction theory [1], the quark wave function is generalized to include an internal part in Section 2.3 characterized by $z_{I}$, and $z_{I I}$ in (1) and (2), which is needed to specify its mass and charge via its flavor. It is shown here that this internal degree freedom plays an analogous role as color does in QCD and "justifies" the assumption of colored quarks, with the difference that quarks in mes- ons have only two "colors". This difference is experimentally testable. QCD is thus reconciled with the scalar strong interaction hadron theory.

\section{REFERENCES}

[1] F. C. Hoh, "Scalar Strong Interaction Hadron Theory," Nova Science Publishers, 2011. https://www.novapublishers.com/catalog/product_info.ph p?products_id=27069

[2] F. C. Hoh, International Journal of Theoretical Physics, Vol. 32, 1993, pp. 1111-1133. doi:10.1007/BF00671793

[3] J. Beringer, et al., Physical Review D, Vol. 86, 2012 Article ID: 010001. doi:10.1103/PhysRevD.86.010001

[4] M. A. B. Bég and H. Ruegg, Journal of Mathematical Physics, Vol. 6, 1965, p. 677 doi:10.1063/1.1704325

[5] F. C. Hoh, Journal of Modern Physics, Vol. 3, 2012, pp. 1562-1571. http://www.scirp.org/journal/jmp

[6] F. C. Hoh, International Journal of Theoretical Physics, Vol. 37, 1998, pp. 1693-1705. doi:10.1023/A:1026640524638

[7] T. D. Lee, "Particle Physics and an Introduction to Field Theory," Harwood Academic Publisher, 1981. 